\title{
Implementing an arts-based intervention for patients with end-stage kidney disease whilst receiving haemodialysis: a feasibility study protocol
}

Claire Carswell $^{1 *}$ D, Joanne Reid ${ }^{1}$, lan Walsh ${ }^{2}$, Helen McAneney ${ }^{2}$ and Helen Noble ${ }^{1}$

\begin{abstract}
Background: End-stage kidney disease is a life-changing illness. Many patients require haemodialysis, a treatment that impacts profoundly on quality of life and mental health. Arts-based interventions have been used in other healthcare settings to improve mental health and quality of life; therefore, they may help address the impact of haemodialysis by improving these outcomes. However, there is a lack of evidence assessing their effectiveness in this population and few randomised controlled trials (RCTs) evaluating the effectiveness of complex arts-based interventions.

Methods: The aims of this study are to establish the feasibility of a cluster RCT of an arts-based intervention for patients with end-stage kidney disease whilst receiving haemodialysis through a cluster randomised pilot study, explore the acceptability of the intervention with a process evaluation and explore the feasibility of an economic evaluation. The study will have three phases. The first phase consists of a cluster randomised pilot study to establish recruitment, participation and retention rates. This will involve the recruitment of 30 participants who will be randomly allocated through cluster randomisation according to shift pattern to experimental and control group. The second phase will be a qualitative process evaluation to establish the acceptability of the intervention within a clinical setting. This will involve semi-structured interviews with 13 patients and three focus groups with healthcare professionals. The third phase will be a feasibility economic evaluation to establish the best methods for data collection within a future cluster RCT.
\end{abstract}

Discussion: Arts-based interventions have been shown to improve quality of life in healthcare settings, but there is a lack of evidence evaluating arts-based interventions for patients receiving haemodialysis. This study aims to assess the feasibility of a future cluster RCT assessing the impact of an arts-based intervention on the wellbeing and mental health of patients receiving haemodialysis and identify the key factors leading to successful implementation. The hope is this study will inform a trial that can influence future healthcare policy by providing robust evidence for arts-based interventions within the haemodialysis setting.

Trial registration: The trial was prospectively registered on clinicaltrials.gov on 14/8/2018, registration number NCT03629496.

Keywords: Art, Feasibility studies, Kidney failure, chronic, Randomised controlled trials as topic, Renal dialysis

\footnotetext{
* Correspondence: ccarswell02@qub.ac.uk

${ }^{1}$ School of Nursing and Midwifery, Queen's University Belfast, 97 Lisburn

Road, Belfast, Northern Ireland

Full list of author information is available at the end of the article
}

(c) The Author(s). 2019 Open Access This article is distributed under the terms of the Creative Commons Attribution 4.0 International License (http://creativecommons.org/licenses/by/4.0/), which permits unrestricted use, distribution, and reproduction in any medium, provided you give appropriate credit to the original author(s) and the source, provide a link to the Creative Commons license, and indicate if changes were made. The Creative Commons Public Domain Dedication waiver (http://creativecommons.org/publicdomain/zero/1.0/) applies to the data made available in this article, unless otherwise stated. 


\section{Background}

End-stage kidney disease is the final stage of chronic kidney disease. It is defined by an estimated glomerular filtration rate of $<15 \mathrm{ml} / \mathrm{min} / 1.73 \mathrm{~m}^{2}$ [1]. The estimated glomerular filtration rate is the estimated rate of fluid filtration within the glomerulus of the kidney and is the main physiological indicator of renal function. Patients with end-stage kidney disease are poly-symptomatic and experience difficult symptoms including fatigue, pruritus, pain, nausea, sexual dysfunction and muscle weakness, which can profoundly impact quality of life (QoL) [2]. The main treatment modalities for end-stage kidney disease are renal replacement therapies, such as haemodialysis. Haemodialysis is a difficult and time-consuming treatment that requires patients to attend hospital up to three times a week for approximately $4 \mathrm{~h}$ each time. During treatment, the patient is connected to a dialysing unit to filter their blood and remove waste products and excess fluid, replacing the role of the kidneys. Patients receiving haemodialysis have lower health-related quality of life (HRQoL) than the general population [3]. Lower HRQoL is associated with increased morbidity and mortality in patients on haemodialysis [4]. Patients receiving haemodialysis also have higher rates of anxiety and depression, with approximately $20-50 \%$ of the patient population experiencing depression and/or anxiety [5-9].

Depression is an independent predictor of mortality in haemodialysis populations [10, 11]; depression may result in poor treatment adherence [6, 12], maladaptive health behaviours [13], self-harm and increased suicide risk [14]. Management of end-stage kidney disease involves an extensive and challenging treatment regimen including compliance with dialysis sessions, medications and fluid and diet restrictions [6]. Non-adherence to such regimens can increase mortality risk-missing a single dialysis session each month increases risk of death by 30\% [15]. Depression is a contributing factor to non-adherence for patients receiving haemodialysis [12]. Anxiety can also have an impact on the physical health of patients receiving haemodialysis, as anxiety symptoms have a significant relationship with performance status $[16,17]$. Therefore, symptoms of both anxiety and depression have a potential impact on the physical wellbeing of patients receiving haemodialysis.

Another collective relationship exists between HRQoL, anxiety and depression in patients receiving haemodialysis. A cross-sectional multi-centre study conducted in Malaysia found that depression, anxiety and stress correlated significantly with HRQoL [8]. Cohort studies within the UK and Ireland also report this association [3]. A systematic literature review found $100 \%$ of identified articles showed a statistically significant relationship between anxiety, depression and HRQoL [18]. Clinical depression influences QoL in varying ways, including impacting mood and motivation $[19,20]$. As previously described, depression can lead to poor treatment adherence [6], which increases symptom burden. Symptoms experienced by patients with end-stage kidney disease impact mood and increase depressive symptoms [2123]. The relationship between HRQoL, anxiety and depression is enmeshed, yet anxiety and depression remains underdiagnosed and under-treated in haemodialysis patients [7, 24-26]. One reason for this complex dynamic is the overlap between anxiety, depression and the uremic state; many symptoms of depression and anxiety, such as anorexia, sleep disturbance and sexual dysfunction, are identical to symptoms of uraemia, making it difficult to differentiate anxiety or depression from the clinical picture of end-stage kidney disease $[5,26]$. Stigma surrounding depression also contributes to diagnosis and treatment rates, as patients are reluctant to agree to psychiatric assessments [27]. HRQoL, anxiety and depression cannot, therefore, be considered in isolation from each other when caring for patients with end-stage kidney disease.

\section{Arts-based interventions}

The application of arts in health has received recent interest because of its' potential to improve patient outcomes and reduce costs for the National Health Service (NHS) [28]. Arts-based interventions involve the implementation of arts activities in a healthcare context to deliver a creative experience [29]. They have been shown to improve QoL, symptom burden and mental health [30-32] in a variety of settings, including the medical-surgical setting [32], primary care [33] and cancer care [34], although there is a dearth of evidence exploring their effect in renal populations. Research examining arts-based interventions is small in scale [35], lacks longitudinal follow-up [36] and focuses on receptive interventions such as music listening $[30,31]$. There are few studies examining the effect of arts-based interventions in patients with end-stage kidney disease; some studies have examined music listening in patients receiving haemodialysis [37], whilst others have explored the use of arts-in-medicine programs. There is a lack of consistent outcome measures in these studies, but there is some evidence that arts-based interventions could have a wide variety of beneficial effects, such as reducing pain and other unpleasant symptoms [38, 39], improving physiological parameters such as oxygen saturation and respiratory rate [39-41], reducing anxiety [42], improving HRQoL [43] and potentially improving biochemical values such as albumin [43]. However, the research exploring arts-in-medicine programs tends to be observational [43] or exploratory $[44,45]$, and no randomised controlled trials have been conducted on non-music listening interventions 
for patients with end-stage kidney disease receiving haemodialysis.

To evaluate the effectiveness of arts-based interventions, rigorous RCTs are necessary. However, there are difficulties associated with RCTs in this arena, particularly around participant recruitment and retention [46]. This is exacerbated in palliative care trials, where participation rates under $50 \%$ are common [47] and trials in nephrology also experience problems retaining participants [48]. Methodology in arts-based intervention research needs to be considered due to the lack of RCTs conducted using non-music interventions [31, 36] and the tendency of complex interventions to fail to demonstrate a statistically significant effect [49]. When evaluating complex interventions, an assessment of the intervention's acceptability for patients and healthcare professionals (HCPs) should be conducted [50]. To explore the acceptability of an intervention, a process evaluation must be conducted. Process evaluations provide an understanding of complex interventions by examining their implementation, mechanism of impact and context [51]. When exploring HCPs' experiences of arts-based interventions, it is also important to examine the impact on HCPs themselves [31, 36]. This includes consideration of potential negative effects of arts-based interventions on the clinical working environment. Arts-based interventions have potential to interfere with work flow, increase stress and restrict communication between HCPs [52]. Any negative consequences could impact patient safety; therefore, the acceptability of an intervention for HCPs is important to consider [53]. The acceptability of the intervention for patients must also be considered. If an effective intervention is burdensome, patients may not participate and not experience any potential benefits [53].

There is also a need to consider arts-based interventions from an economic perspective, as current guidance on research into arts-based interventions recommends cost-effectiveness or cost-utility is evaluated [54], as the National Institute for Health and Care Excellence (NICE) use cost-effectiveness to inform recommendations for funding healthcare interventions [55]. Whilst there is evidence that suggests arts-based interventions can save the healthcare system money (arts-on-prescription are thought the save the NHS $£ 216$ per person) [35], there is a lack of formal economic evaluations within the evidence base [56]. Consequently, it is important to explore the best methods for collecting the data needed for future analysis, to ensure that a formal economic evaluation is feasible within an RCT.

\section{Objectives}

- To assess the feasibility of conducting a definitive randomised controlled trial to evaluate the impact of an arts-based intervention through a cluster ra ndomised pilot study.

- To explore the acceptability of the implementation of an arts-based intervention and randomised controlled trial in a haemodialysis unit for both patients and HCPs.

- To assess the feasibility of conducting a definitive economic evaluation of an arts-based intervention for patients receiving haemodialysis.

\section{Methods}

\section{Research design}

The study will consist of three phases and will utilise a parallel mixed-method design. Phase 1 will involve a quantitative cluster randomised pilot study, phase 2 is a qualitative process evaluation and phase 3 is a feasibility economic evaluation [57].

\section{Research setting}

The research will be conducted in an outpatient haemodialysis unit of a teaching hospital in the Northern Ireland. The unit provides haemodialysis to approximately 120 patients. Participants will be recruited from the unit using convenience sampling.

\section{Cluster randomised pilot study Participants}

Eligibility criteria for patients:

- Age 18 or over

- Able and willing to provide consent

- Receiving haemodialysis (both incident and prevalent haemodialysis patients will be eligible)

There is little consensus on the appropriate sample size for a feasibility study, with guidance ranging from 12 per arm [58] to 50 per arm [59]. This guidance reflects the inconsistent reporting of feasibility studies. A review of pilot and feasibility studies concluded studies were poorly reported and placed an inappropriate emphasis on hypothesis testing [60]. National Institute of Health Research published guidelines highlighting hypothesis testing is inappropriate in feasibility studies [61]; therefore, a power calculation has not been conducted, as hypothesis testing is not an objective of this trial. A sample size of 30 is recommended by the National Institute for Health Research (NIHR) [62] for parameter estimation within an RCT. A university statistician confirmed that a sample of 30 was appropriate to satisfy the research objectives. 


\section{Recruitment}

Eligible patients will be screened by a gatekeeper working in the haemodialysis unit. Screening logs will be used to measure the proportion of patients who are eligible, who are interested in participating and reasons for non-participation [63]. Patients who are eligible will be asked to consent to a first approach by the researcher, who will describe the study and provide the patient with a participant information sheet. The researcher will allow each patient a period of $48 \mathrm{~h}$ to consider participation before providing a standardised consent form.

\section{Randomisation}

Participants will be randomly allocated to the control group, receiving usual care, or the experimental group, receiving usual care plus the arts-based intervention. The control group will be asked not to participate in visual art or creative writing during haemodialysis sessions until data collection is complete. Once data collection has been completed, the control group will receive the same art materials as the intervention group and a facilitated session of art activities whilst on haemodialysis.

Cluster randomisation according to shift pattern (Monday, Wednesday, Friday or Tuesday, Thursday, Saturday) will be used, as this reduces the risk of contamination of the control group. Any identified instances of contamination or deviations from protocol, such as patients attending different shifts and being exposed to the intervention, will be documented in order to inform the best randomisation strategy within a definitive trial. Researchers will aim to recruit an equal number of participants from each shift to ensure both the control and experimental groups contain an equal number of participants [64]. The randomisation procedure will be carried out by a member of staff within the university who is not affiliated with the study. They will toss a coin which will determine what shift pattern will receive the intervention. The allocation will be placed in a sealed envelope that will be opened by the researcher once baseline data collection has been completed.

\section{Intervention}

The intervention is based on the psychological theory of flow, which posits the existence of a 'flow state', a state of optimal experience that results from complete absorption in a task. In order to induce a flow state, the task must present a challenge to the individual that they can overcome through the development of their skills $[65,66]$. Qualitative literature has suggested arts-in-medicine programmes, person-centred arts programmes that are delivered within hospitals, can induce the hallmark experiences of a flow state in patients who participate, such as an altered perception of time and reduction in rumination and anxiety $[43,45,67]$. Therefore, the intervention has been modelled on these programmes, with additional structure put in place to allow for assessment of dose and fidelity.

The intervention consists of six 1-hourly art sessions, implemented at the bedside whilst the participant is receiving haemodialysis and facilitated by the researcher. Each participant will receive the sessions over a course of 3 weeks, receiving two sessions a week. This time frame was chosen in consultation with the study's interdisciplinary advisory group who recommended each participant receive a day off each week to reduce the potential of fatigue influencing participation, in consultation with experts in the field of arts in health who established six sessions was an adequate dose [68], and reviewing previous literature that identified $1 \mathrm{~h}$ is the optimal time frame for implementing an art activity [69]. The activities on offer will consist of a selection of discrete choices, either creative writing and visual art, but will involve a person-centred approach that will allow patients to adapt the activities to their interests and their abilities. This person-centred approach is modelled on the arts-in-medicine programmes that have shown evidence of being sustainable in clinical settings over prolonged periods of time [43-45].

Each participant will receive their own individual arts pack that will contain a standardised set of materials that were selected by the study's interdisciplinary advisory group. The items were selected according to their ease of use and their ability to be implemented without impacting the clinical setting. Each participant will receive:

- Sketch book

- Graphite pencils

- Graphic pens

- Watercolour paints

- Watercolour brush pen with in-built water container

- Colouring pencils

- Drawing board

- Drawing board clip

- Eraser

- Sharpener

- Pencil grip

Individual packs will be provided to each participant to maintain infection control and reduce any issue of cross-contamination between participants. All participants will keep the packs at the end of the study. Each session will involve one to one facilitation to ensure the activities are accessible for the majority of 
patients, as arteriovenous fistulas and problems with dexterity can limit a person's ability to use the materials unassisted. Patient preference of activity, materials and engagement will be captured in activity logs by the researcher implementing the intervention.

\section{Data collection and management}

Feasibility measures The primary outcome of the trial will be the recruitment rate of participants within a single site. Assessing the ability to recruit and retain participants is a common issue explored in feasibility trials and randomised pilot studies [60, 63, 70, 71]. Reasons for non-participation will be collected in screening logs during the recruitment phase. Previous experience with art will be collected in these screening logs to explore whether experience influences recruitment or retention of participants.

Secondary outcomes include the attrition rate of participants over a period of 3 months to assess the feasibility of longitudinal follow-up within a definitive trial. The acceptability of outcome measures will also be assessed by completion rates of clinical outcome measures, missing data, and will be further informed by the parallel process evaluation.

Clinical outcome measures Baseline demographic and clinical data will include age, gender, ethnicity, education, dialysis vintage, frailty and co-morbidities; this will be collected through self-report to establish any differences between control and experimental groups and to identify any factors that may contribute to participation or attrition within the trial.

Due to the wide variety of primary outcomes evaluated in the existing evidence base, it is not clear what the most appropriate primary clinical outcome measure is for a definitive trial; however, assessment of the appropriateness of clinical outcome measures and exploration of potential mechanisms of impact can be explored during feasibility testing to ensure the most relevant clinical outcomes are included in a definitive trial [72]. The most consistently reported improvement in the existing literature base is anxiety [73]; therefore, the Hospital Anxiety and Depression Scale (HADS) will be used; this scale has been validated in patients with end-stage kidney disease [74] and enables exploration of both depression and anxiety independently through a subscale analysis [75].

The Kidney Disease Quality of Life Short Form 36 (KDQoL-SF36) will be used to measure HRQoL; this is a commonly used tool in renal literature, is both valid and reliable and provides the most comprehensive overview of contributing factors to QoL [76]. This questionnaire was selected as it is the most comprehensive HRQoL scale with the ability to explore different subscales [76], and observational studies have suggested that arts-based interventions may result in improvements in the KDQoL-SF 36, improve physiological parameters and help reduce unpleasant physical symptoms of the disease [38, 39, 43, 77].

Arts-based intervention research faces criticism for lack of longitudinal follow-up, which is necessary to assess sustainability and lasting benefits [36]. Participants who are lost to follow-up in longitudinal RCTs of complex interventions tend to be older, diagnosed with a chronic illness and have higher co-morbidity [78], common demographic factors in patients with end-stage kidney disease [79]. Therefore, to establish feasibility of longitudinal follow-up according to attrition rates and assess the impact of participant burden, clinical outcome measures will be collected at baseline, immediately post-intervention, at 6 weeks and 3 months. The follow-up time points within this feasibility study will allow exploration of reasons for attrition and provide participants with an opportunity to familiarise themselves with the outcome measures and assess participant burden for further exploration within the process evaluation.

The schedule for enrolment, administration of interventions and assessment of outcomes (including those for phase 2 and 3) can be seen in Fig. 1.

\section{Data analysis}

Data analysis will be conducted using the Statistical Package for the Social Sciences (SPSS v 24). Descriptive statistics will be used to present baseline demographic and clinical data. Categorical data will be presented as frequencies and percentages, whilst continuous data will be presented as means and standard deviations. Recruitment, participation and retention rates will be reported and presented in a CONSORT flow diagram [80]. The proportion of patients who were eligible for recruitment, who consented to participate and who completed the study will be presented with $95 \%$ confidence intervals.

Exploratory inferential statistics will be conducted to establish the time needed for data analysis and to explore any potential effects from clustering at the level of shift pattern, but no conclusions of effectiveness of the intervention will be made. Differences in baseline and demographic measures between the clusters will be compared using analysis of variance (ANOVA) for continuous data and $\chi^{2}$ for categorical data to identify any unanticipated confounding variables introduced by clustering according to shift pattern. As the study is not statistically powered, the results will be interpreted with caution. Independent $t$ tests (or Mann-Whitney $U$ ) will be conducted to compare the average scores of the experimental group 


\begin{tabular}{|c|c|c|c|c|c|c|}
\hline \multirow[b]{3}{*}{ TIMEPOINT $^{\star *}$} & \multicolumn{6}{|c|}{ STUDY PERIOD } \\
\hline & \multirow{2}{*}{$\begin{array}{c}\text { Enrolment } \\
-t_{1}\end{array}$} & \multirow{2}{*}{$\frac{\text { Allocation }}{0}$} & \multicolumn{3}{|c|}{ Post-allocation } & \multirow{2}{*}{$\frac{\text { Close-out }}{t_{x}}$} \\
\hline & & & $\begin{array}{c}3 \text { weeks } \\
t_{1}\end{array}$ & $\begin{array}{c}6 \text { weeks } \\
t_{2}\end{array}$ & $\begin{array}{c}3 \text { months } \\
t_{3}\end{array}$ & \\
\hline \multicolumn{7}{|l|}{ ENROLMENT: } \\
\hline \multirow{3}{*}{$\begin{array}{r}\text { Eligibility screen } \\
\text { Informed consent } \\
\text { Allocation }\end{array}$} & $x$ & & & & & \\
\hline & $\mathrm{x}$ & & & & & \\
\hline & & $\mathrm{x}$ & & & & \\
\hline \multicolumn{7}{|l|}{ INTERVENTIONS: } \\
\hline \multicolumn{7}{|l|}{6 art sessions } \\
\hline $\begin{array}{r}\text { Single art session } \\
\text { (control) }\end{array}$ & & & & & & $x$ \\
\hline \\
\hline \multicolumn{7}{|l|}{$\begin{array}{l}\text { Baseline: Age, } \\
\text { gender education, } \\
\text { dialysis vintage, } \\
\text { ethnicity, CFS, co- } \\
\text { morbidities }\end{array}$} \\
\hline \multicolumn{7}{|l|}{ PHASE 1} \\
\hline Recruitment rates & $x$ & & & & & $x$ \\
\hline Attrition rates & & $x$ & $x$ & $x$ & $x$ & $x$ \\
\hline \multirow{2}{*}{$\begin{array}{r}\text { KDQOL-SF } 36 \\
\text { HADS }\end{array}$} & & $x$ & $x$ & $x$ & $x$ & \\
\hline & & $x$ & $x$ & $x$ & $x$ & \\
\hline \multicolumn{7}{|l|}{$\begin{array}{r}\text { Qualitative semi- } \\
\text { structured interviews } \\
\text { with participants }\end{array}$} \\
\hline \multicolumn{7}{|l|}{$\begin{array}{r}\text { Focus groups with } \\
\text { HCP }\end{array}$} \\
\hline \multicolumn{7}{|l|}{ PHASE 3} \\
\hline \multirow{2}{*}{$\begin{array}{r}E Q-5 D-5 L \\
\text { Patient resource use } \\
\text { log }\end{array}$} & & $x$ & $x$ & $x$ & $x$ & \\
\hline & & $x$ & $x$ & $x$ & $x$ & \\
\hline
\end{tabular}

Fig. 1 SPIRIT figure illustrating the schedule of enrolment for participants, implementation of interventions and timeline of assessments for phases 1,2 and 3

and control group. The majority of arts-based intervention research involves pre- and post-test designs; therefore, a repeated measures $t$ test (or Wilcoxon matched pairs test) will also be conducted.

\section{Process evaluation \\ Participants}

Participants will include patients and HCPs. The patients recruited will include those who either participated or withdrew from the cluster randomised pilot study. HCPs from the unit will also be recruited into the process evaluation.

Eligibility criteria for HCPs:

- A member of the multidisciplinary healthcare team, including nurses, healthcare support workers, doctors, dietitians, social workers and counsellors who:

- Has had exposure to the intervention.
- Has worked in a renal setting for more than 3 months

Familiarity with the context of the clinical environment is needed to inform the acceptability of the intervention [53]. Context includes the social system of the work place, taking into consideration social norms and material resources [53].

\section{Recruitment}

During the cluster randomised pilot study, patients who have been recruited will be offered the opportunity to participate in the process evaluation. A separate information sheet and consent form will be provided for this phase. Participants from both the intervention and control group will be recruited, and participants will be informed that participation in the process evaluation is not dependent on completion of the cluster randomised pilot study, so participants 
who decide to withdraw from the feasibility study can be included in the sample.

HCPs will be recruited for the process evaluation by purposive sampling. During the feasibility trial, the researcher implementing the intervention will identify HCPs who are present on the unit during implementation of the intervention. The unit manager, acting as the gatekeeper, will screen the identified HCPs to ensure they meet the inclusion criteria and it is acceptable for the researcher to make the initial approach. Due to managerial and social hierarchies within hospitals, HCPs may feel pressure to participate; therefore, HCPs will be approached by the researcher directly, so the gatekeeper will be unaware of who is participating.

\section{Data collection and management}

Approximately 13 patients will be recruited into the process evaluation. The principle of $10+3$ for data saturation outlines a minimum of 10 interviews should be conducted, followed by at least three consecutive interviews that present no new findings [81]. Three focus groups will be conducted with five HCPs per focus group [82, 83]. The dialysis unit in which the study is taking place is small, so the focus group size will be limited by the HCPs available $[84,85]$. Data collection will continue until data saturation is reached.

Data collection for the qualitative process evaluation will occur parallel to the cluster randomised pilot study, as this allows adaptation of the intervention to identified concerns during the feasibility stage, as recommended by the Medical Research Council (MRC) guidance [50]. The semi-structured interviews will be conducted with patients in their own home to avoid the power dynamic present in a clinical setting [86]. This approach will be flexible and patients can request a different location. The focus groups with HCPs will take place in a location convenient for all participants. The process evaluation will follow MRC guidance [50] and will focus on the acceptability of the intervention for patients and HCPs, and the interview guides will be informed by the RE-AIM QuEST framework [87]. The interview guide for the semi-structured interviews for patients and focus groups for HCPs can be found in Additional file 1 .

\section{Data analysis}

The semi-structured interviews and focus groups will be conducted by the same researcher and recorded and transcribed verbatim. Inductive thematic analysis will be used to analyse the data collected. Thematic analysis involves identifying and coding central themes within qualitative data through an iterative process [88]. Investigator triangulation will be used to ensure validity of the identified themes [89].

\section{Economic evaluation Data collection and management}

Feasibility studies are not adequately powered to establish effectiveness; therefore, conducting a cost-utility evaluation is not appropriate [90]. However, the feasibility of data collection methods and appropriateness of outcome measures will be evaluated during feasibility testing to inform an economic evaluation within a definitive RCT.

Health and social care costs of participants will be collected using a Patient Service Use Log, a prospective $\log$ that captures information on participants healthcare resource use during their time in the study [91]. These will be provided to participants during baseline data collection of the cluster randomised pilot study and collected during follow-up, post-intervention and at 6 weeks and 3 months by the researcher. The outcome of interest is the completion rate for the Patient Service Use Log. The EQ-5D-5 L will be administered to patients in conjunction with clinical outcome measures, pre-/post-intervention and at 6 weeks and 3 months follow-up. The outcome of interest is the acceptability of the EQ-5D-5 L for use within an economic evaluation, as determined by completion rates, missing data and assessment within the process evaluation. The EQ-5D-5 L is recommended by NICE for deriving utility values for the calculation of quality-adjusted life years in economic evaluations [92]. Whilst it is not anticipated that arts-based interventions can improve all items of the EQ-5D-5 L, there is evidence that suggests they may help patients manage pain [38], improve dexterity [93], reduce depression and anxiety [94] and subsequently improves motivation and engagement in daily activities [31, 95, 96].

\section{Data analysis}

A cost-consequence analysis is recommended by the NIHR for feasibility studies [97]. Costs and outcome measures will be presented using descriptive statistics, including means and $95 \%$ confidence intervals to show a general overview of the economic consequences of the intervention. Completion rates and missing data for the Patient Service Use Log and the EQ-5D-5 L will be presented as frequencies to assess the feasibility of data collection.

\section{Progression criteria}

Progression to a definitive RCT will be determined by recruitment rates [98] and the acceptability of the intervention for patients and staff. Acceptability will be 
evaluated through the qualitative process evaluation, as it is recommended that both quantitative and qualitative data are used when determining the feasibility of a randomised controlled trial [50, 72]:

- $75-100 \%$ of the target sample size recruited from a single site will result in progression to a definitive RCT.

- $50-74 \%$ of the target sample size recruited from a single site will result in progression to a definitive trial after reviewing the protocol and data from the process evaluation, and making appropriate amendments to address barriers to recruitment.

- $25-49 \%$ of the target sample size recruited from a single site will result in progression to a definitive trial after reviewing the protocol with input from potential co-applicants to ensure that the protocol is modified to enhance recruitment rates.

- Less than $25 \%$ of the target sample size recruited from a single site will probably result in the trial not progressing, unless a significant modifiable barrier is identified within the process evaluation.

- More than 20\% attrition rate [99] from the recruited sample will result in revision of the protocol and data from the process evaluation, and appropriate amendments will need to be made to address barriers to retention of participants, prior to progression to a full trial.

- Progression to a full trial will be contingent on the acceptability of the intervention for both patients and HCPs regardless of recruitment rates. This will be explored within the qualitative process evaluation [87]. Any necessary modifications identified will be made prior to progression to a definitive trial.

- The use of the outcome measures in a definitive trial will be contingent on the acceptability of the questionnaires, which will be informed by completion rates and through the qualitative process evaluation. Any necessary modifications, such as removing questionnaires or using only certain subscales, will be made prior to progression to a definitive trial.

\section{Discussion}

Whilst patients with end-stage kidney disease receiving haemodialysis may stand to benefit from arts-based interventions, the lack of RCTs limits the ability of the evidence base to influence healthcare policy and enable access to these interventions. Arts-based interventions also constitute a complex intervention according to MRC guidance, and therefore, additional factors outside of efficacy, including the context of implementation, acceptability for patients and acceptability for HCPs, also need to be considered to inform optimal strategies for implementation in clinical practice. This article has outlined a protocol to develop an arts-based intervention for patients with end-stage kidney disease whilst receiving haemodialysis and to assess the feasibility of a parallel cluster randomised controlled trial and economic evaluation. This study will provide a foundation from which a definitive RCT protocol can be developed that can assess the effectiveness of highly complex arts-based interventions for patients with end-stage kidney disease receiving haemodialysis, within a framework that can be used to inform healthcare policy.

\section{Limitations}

One limitation of this protocol is the lack of differentiation of roles. As a consequence of limited resources, the development of the intervention, the implementation of the intervention, data collection and data analysis will be conducted by a single research team. Ideally, these roles would be distinct and performed by separate teams, as outlined in the MRC guidelines [50]. Any future definitive trial should have separate teams implementing the intervention, collecting the quantitative data and collecting the qualitative data for a process evaluation to reduce the risk of bias. It is also recommended that in any future process evaluation, multiple team members should transcribe and thematically analyse qualitative data. Another limitation is the lack of attention placebo provided to the control group [100]; whilst no hypothesis testing is being conducted to establish the efficacy of the intervention, attention placebo control may influence retention of participants in the comparator group. Implementation of two different interventions is not feasible within the restricted resources of this study; however, the process evaluation may help inform the elements needed in a future attention placebo control group within a definitive RCT. An additional consideration that will inform a definitive RCT is the need for multiple clusters to control for between-cluster variation. Whilst the size of this study restricted randomisation to only two clusters, a definitive trial will likely require multiple sites to ensure an adequate number of clusters.

\section{Additional file}

Additional file 1: Process evaluation interview guide for experimental group. (DOCX $21 \mathrm{~kb})$

\section{Abbreviations}

HCP: Healthcare professional; HRQOL: Health-related quality of life; MRC: Medical Research Council; NHS: National Health Service; NICE: National Institute for Health and Care Excellence; NIHR: National Institute for Health Research; QoL: Quality of life; RCT: Randomised controlled trial

\section{Acknowledgements}

The authors would like to thank the members of the study's interdisciplinary advisory group for their support during the development of the protocol. 


\section{Funding}

This research is funded by a Department for the Economy PhD studentship. The funder has had no input relating to the design of the study, the collection, analysis or interpretation of data, or for the writing of the manuscript. Additional funding has also been obtained following completion of the protocol from Kidney Care UK and Northern Ireland Kidney Patient Association. This funding is to purchase the art materials required to implement the intervention.

\section{Availability of data and materials}

Data sharing is not applicable to this article as no datasets have been generated or analysed. The data generated during the study described in the protocol will be made available on clinicaltrials.gov as per the requirements for registration. Clinicaltrials.gov number is NCT03629496.

\section{Authors' contributions}

CC, HN, JR and IW are involved in developing the trial design and writing and drafting of the study protocol. HMCA is involved in the trial design, specifically relating to the cluster randomised pilot study and statistical analysis, and drafting of the study protocol. All authors read and approved the final manuscript.

\section{Author's information}

The author CC is a current PhD student at Queen's University Belfast in Northern Ireland and will be undertaking this study as their PhD project, while HN, JR and IW are members of their supervisory team. Consequently the recruitment, implementation of the intervention, data collection for the cluster randomised pilot study, economic evaluation and process evaluation, and analysis of collected data, will all be completed by CC. Ideally these roles would be performed by different people or research teams to prevent introduction of bias, such as the halo effect, but the constraints of a PhD project do not allow this.

\section{Ethics approval and consent to participate}

This study has obtained ethical approval from the Office of Research Ethics Committees Northern Ireland (Ref: 18/NI/0091). An informed consent form will be signed by patients participating in the cluster randomised pilot study and parallel feasibility economic evaluation. A separate consent form will also be signed by patients and healthcare staff who participate in the parallel process evaluation.

\section{Consent for publication}

Not applicable

\section{Competing interests}

The authors declare that they have no competing interests.

\section{Publisher's Note}

Springer Nature remains neutral with regard to jurisdictional claims in published maps and institutional affiliations.

\section{Author details}

'School of Nursing and Midwifery, Queen's University Belfast, 97 Lisburn Road, Belfast, Northern Ireland. ${ }^{2}$ School of Medicine, Dentistry and Biomedical Sciences, Queen's University Belfast, Belfast, Northern Ireland.

Received: 21 August 2018 Accepted: 19 December 2018 Published online: 05 January 2019

\section{References}

1. National Institute for Health and Care Excellence. Chronic kidney disease in adults: assessment and management | Guidance and guidelines | NICE. 2014. https:// unw.nice.org.uk/guidance/cg182/chapter/1-Recommendations\#classification-ofchronic-kidney-disease-2. Accessed 10 Nov 2017.

2. Raj R, Ahuja KDK, Frandsen M, Jose M. Symptoms and their recognition in adult haemodialysis patients: interactions with quality of life. Nephrology. 2017;22:228-33.

3. Lowney AC, Myles HT, Bristowe K, Lowney EL, Shepherd K, Murphy M, et al. Understanding what influences the health-related quality of life of hemodialysis patients: a collaborative study in England and Ireland. J
Pain Symptom Manag. 2015;50:778-85. https://doi.org/10.1016/j. jpainsymman.2015.07.010

4. Liebman S, Li N-C, Lacson E. Change in quality of life and one-year mortality risk in maintenance dialysis patients. Qual Life Res. 2016;25:2295-306. https://doi.org/10.1007/s11136-016-1257-y.

5. Cohen SD, Norris L, Acquaviva K, Peterson RA, Kimmel PL. Screening, diagnosis, and treatment of depression in patients with end-stage renal disease. Clin J Am Soc Nephrol. 2007;2:1332-42.

6. Nabolsi MM, Wardam L, Al-Halabi JO. Quality of life, depression, adherence to treatment and illness perception of patients on haemodialysis. Int J Nurs Pract. 2015;21:1-10.

7. Kokoszka A, Leszczyńska K, Radzio R. Prevalence of depressive and anxiety disorders in dialysis patients with chronic kidney disease. Arch Psychiatry Psychother. 2016;1:8-13.

8. Bujang MA, Musa R, Liu WJ, Chew TF, Lim CTS, Morad Z. Depression, anxiety and stress among patients with dialysis and the association with quality of life. Asian J Psychiatr. 2015;18:49-52. https://doi.org/10.1016/j.ajp.2015.10.004.

9. Goh ZS, Griva K. Anxiety and depression in patients with end-stage renal disease: impact and management challenges - a narrative review. Int J Nephrol Renovasc Dis. 2018;11:93-102.

10. Chilcot J, Davenport A, Wellsted D, Firth J, Farrington K. An association between depressive symptoms and survival in incident dialysis patients. Nephrol Dial Transplant. 2011;26:1628-34.

11. Barros A, da Costa BE, Mottin CC, D'Avila DO. Depression, quality of life, and body composition in patients with end-stage renal disease: a cohort study. Rev Bras Psiquiatr. 2016;38:301-6.

12. Ossareh S, Tabrizian S, Zebarjadi M. Prevalence of depression in maintenance hemodialysis patients and its correlation with adherence to medications. Iran J Kidney Dis. 2014;8:467-75 http://www.ncbi.nlm.nih.gov/ pubmed/25362222.

13. Ziegelstein RC, Fauerbach J a, Stevens SS, Romanelli J, Richter DP, Bush $D E$. Patients with depression are less likely to follow recommendations to reduce cardiac risk during recovery from a myocardial infarction. Arch Intern Med. 2000;160:1818-23.

14. Pompili $\mathrm{M}$, Venturini $\mathrm{P}$, Montebovi F, Forte A, Palermo M, Lamis $\mathrm{D}$ a, et al. Suicide risk in dialysis: review of current literature. Int J Psychiatry Med. 2013;46:85-108. https://doi.org/10.2190/PM.46.1.f.

15. Denhaerynck K, Manhaeve D, Dobbels F, Garzoni D, Nolte C, De Geest S. Prevalence and consequences of nonadherence to hemodialysis regimens. Am J Crit Care. 2007:16:222-35.

16. Loosman WL, Rottier MA, Honig A, Siegert CEH. Association of depressive and anxiety symptoms with adverse events in Dutch chronic kidney disease patients: a prospective cohort study. BMC Nephrol. 2015; 16:1-8. https://doi.org/10.1186/s12882-015-0149-7.

17. Rajan EE, Subramanian $\mathrm{S}$. The effect of depression and anxiety on the performance status of end-stage renal disease patients undergoing hemodialysis. Saudi J Kidney Dis Transplant. 2016;27:331. https://doi.org/ 10.4103/1319-2442.178555.

18. García-Llana H, Remor E, del Peso G, Selgas R. El papel de la depresión, la ansiedad, el estrés y la adhesión al tratamiento en la calidad de vida relacionada con la salud en pacientes en diálisis: Revisión sistemática de la literatura. Nefrologia. 2014;34:637-57.

19. Mccann K, Bsc R, Boore JRP, Rn O, Rnt R, Frcn BP, et al. Fatigue in persons with renal failure who require maintenance haemodialysis. J Adv Nurs. 2000;32:1132-42.

20. Farragher JF, Polatajko HJ, Jassal SV. The relationship between fatigue and depression in adults with end-stage renal disease on chronic in-hospital hemodialysis: a scoping review. J Pain Symptom Manag. 2017;53:783-803. e1. https://doi.org/10.1016/j.jpainsymman.2016.10.365.

21. Cao X, Tian L, Lin C. Symptom clusters in patients receiving haemodialysis: a systematic review of observational studies. J Clin Nurs. 2017;26:2545-57. https://doi.org/10.1111/jocn.13644.

22. Maung S, El Sara A, Cohen D, Chapman C, Saggi S, Cukor D. Sleep disturbance and depressive affect in patients treated with haemodialysis. Ren Care. 2017;43:60-6.

23. Wan Zukiman WZH, Yaakup H, Zakaria NF, SA Bin S. Symptom prevalence and the negative emotional states in end-stage renal disease patients with or without renal replacement therapy: a cross-sectional analysis. J Palliat Med. 2017;20:1127-34. https://doi.org/10.1089/jpm.2016.0450.

24. Watnick S, Kirwin P, Mahnensmith R, Concato J. The prevalence and treatment of depression among patients starting dialysis. Am J Kidney Dis. 2003;41:105-10. 
25. Cukor D, Coplan J, Brown C, Peterson RA, Kimmel PL. Course of depression and anxiety diagnosis in patients treated with hemodialysis: a 16-month follow-up. Clin J Am Soc Nephrol. 2008:3:1752-8.

26. Cohen SD, Cukor D, Kimmel PL. Anxiety in patients treated with hemodialysis. Clin J Am Soc Nephrol. 2016;11:2250-5.

27. Wuerth D, Finkelstein SH, Finkelstein FO. Psychosocial factors in patients with chronic kidney disease: the identification and treatment of depression in patients maintained on dialysis. Semin Dial. 2008;18:142-6. https://doi. org/10.1111/j.1525-139X.2005.18213.x

28. All-Party Parliamentary Group on Arts Health and Wellbeing. Creative health: the arts for health and wellbeing | The short report. 2017.

29. Centre for Arts in Medicine. Talking about arts in health: a white pape addressing the language used to describe the discipline from a higher education perspective. 2017:;1-24. http://arts.ufl.edu/site/assets/files/106496/ uf_cam_language_whitepaper.pdf.

30. Staricoff R, Clift S. Arts and music in healthcare: an overview of the medical literature: 2004-2011. Chelsea and Westmister Health Charity; London; 2011.

31. Bungay $H$, Munn-Giddigs $C$, Wilson $C$. The value of the arts in therapeutic and clinical interventions: a critical review of the literature. Arts \& Humanities Research Council: Cambridge; 2014.

32. Sonke J, Pesata V, Arce L, Carytsas FP, Zemina K, Jokisch C. The effects of arts-in-medicine programming on the medical-surgical work environment. Arts Heal. 2015;7:27-41.

33. Crone DM, O'Connell EE, Tyson PJ, Clark-Stone F, Opher S, James DVB. It helps me make sense of the world: the role of an art intervention for promoting health and wellbeing in primary care-perspectives of patients, health professionals and artists. J Public Heal. 2012;20:519-24.

34. Kim KS, Loring S, Kwekkeboom K. Use of art-making intervention for pain and quality of life among cancer patients: a systematic review. J Holist Nurs. 2017;36:1-13. https://doi.org/10.1177/0898010117726633 .

35. All-Party Parliamentary Group on Arts Health and Wellbeing. The arts for health and wellbeing | Inquiry report. 2017.

36. Boyce $M$, Bungay $H$, Munn-Giddings $C$, Wilson $C$. The impact of the arts in healthcare on patients and service users: a critical review. Heal Soc Care Community. 2017;26:1-16.

37. Huang S-T, Good M, Zauszniewski JA. The effectiveness of music in relieving pain in cancer patients: a randomized controlled trial. Int J Nurs Stud. 2010; 47:1354-62. https://doi.org/10.1016/j.jinurstu.2010.03.008.

38. Shabandokht-Zarmi H, Bagheri-Nesami M, Shorofi SA, Mousavinasab SN. The effect of self-selected soothing music on fistula puncture-related pain in hemodialysis patients. Complement Ther Clin Pract. 2017;29:53-7. https:// doi.org/10.1016/j.ctcp.2017.08.002.

39. Burrai F, Micheluzzi V, Zito MP, Pietro G, Sisti D. Effects of live saxophone music on physiological parameters, pain, mood and itching levels in patients undergoing haemodialysis. J Ren Care. 2014;40:249-56. https://doi. org/10.1111/jorc.12078

40. Lin Y-J, Lu K-C, Chen C-M, Chang C-C. The effects of music as therapy on the overall well-being of elderly patients on maintenance hemodialysis. Biol Res Nurs. 2012;14:277-85. https://doi.org/10.1177/1099800411413259.

41. Hou Y-C, Lin Y-J, Lu K-C, Chiang H-S, Chang C-C, Yang L-K. Music therapyinduced changes in salivary cortisol level are predictive of cardiovascular mortality in patients under maintenance hemodialysis. Ther Clin Risk Manag. 2017;13:263-72. https://doi.org/10.2147/TCRM.S127555.

42. Salehi B, Salehi M, Nsirnia K, Soltani P, Adalatnaghad M, Kalantari N, et al. The effects of selected relaxing music on anxiety and depression during hemodialysis: a randomized crossover controlled clinical trial study. Arts Psychother. 2016;48:76-80

43. Ross EA, Hollen TL, Fitzgerald BM. Observational study of an arts-inmedicine program in an outpatient hemodialysis unit. Am J Kidney Dis. 2006:47:462-8. https://doi.org/10.1053/j.ajkd.2005.11.030.

44. Corrigan C, Peterson L, McVeigh C, Lavin P, Mellotte G, Baker Kerrigan A, et al. The perception of art among patients and staff on a renal dialysis unit. Ir Med J. 2017;110:633 http://imj.ie/the-perception-of-art-among-patients-andstaff-on-a-renal-dialysis-unit/. Accessed 27 Nov 2017.

45. Rowe N, Jones $\mathrm{CH}$, Seeger L, Greaves G, Holman C, Turner H. Forgetting the machine: patients ' experiences of engaging in artwork while on renal dialysis. J Appl Arts Heal. 2011;2:57-72.

46. Donovan JL, De Salis I, Toerien M, Paramasivan S, Hamdy FC, Blazeby $J M$. The intellectual challenges and emotional consequences of equipoise contributed to the fragility of recruitment in six randomized controlled trials. J Clin Epidemiol. 2014;67:912-20. https://doi.org/10. 1016/j.jclinepi.2014.03.010

47. Hanson LC, Bull J, Wessell K, Massie L, Bennett RE, Kutner JS, et al. Strategies to support recruitment of patients with life-limiting illness for research: the palliative care research cooperative group. J Pain Symptom Manag. 2015;48:1021-30.

48. Palmer SC, Sciancalepore M, Strippoli GFM. Trial quality in nephrology: how are we measuring up? Am J Kidney Dis. 2011;58:335-7. https://doi.org/10. 1053/j.ajkd.2011.06.006.

49. Levati S, Campbell P, Frost R, Dougall N, Wells M, Donaldson C, et al. Optimisation of complex health interventions prior to a randomised controlled trial: a scoping review of strategies used. Pilot Feasibility Stud. 2016;2:17. https://doi.org/10.1186/s40814-016-0058-y.

50. Moore G, Audrey S, Barker M, Bond L, Bonell C, Hardeman W, et al. Process evaluation of complex interventions: Medical Research Council guidance. BMJ. 2015;350:h1258.

51. Moore GF, Audrey S, Barker M, Bond L, Bonell C, Hardeman W, et al. Process evaluation of complex interventions: Medical Research Council guidance. BMJ. 2015;350:h1258. https://doi.org/10.1136/bmj.h1258.

52. Wilson C, Bungay H, Munn-Giddings C, Boyce M. Healthcare professionals' perceptions of the value and impact of the arts in healthcare settings: a critical review of the literature. Int J Nurs Stud. 2015;56:90-101. https://doi. org/10.1016/j.ijnurstu.2015.11.003.

53. May C. Towards a general theory of implementation. Implement Sci. 2013;8: 18. https://doi.org/10.1186/1748-5908-8-18.

54. Fancourt D, Joss T. Aesop: a framework for developing and researching arts in health programmes. Arts \& Health. 2015;7:1-13. https://doi.org/10.1080/ 17533015.2014 .924974

55. Ogden J. QALYS and their role in the NICE decision-making process. Prescriber. 2017;28:41-3. https://doi.org/10.1002/psb.1562.

56. Craemer R. The arts and health: from economic theory to cost-effectiveness. Univ Melb Ref e-Journal. 2009:1:1-14.

57. Eldridge SM, Lancaster GA, Campbell MJ, Thabane L, Hopewell S, Coleman $\mathrm{CL}$, et al. Defining feasibility and pilot studies in preparation for randomised controlled trials: development of a conceptual framework. PLoS One. 2016:11:1-22.

58. Julious SA. Sample size of 12 per group rule of thumb for a pilot study. Pharm Stat. 2005:4:287-91.

59. Sim J, Lewis $M$. The size of a pilot study for a clinical trial should be calculated in relation to considerations of precision and efficiency. J Clin Epidemiol. 2012;65:301-8. https://doi.org/10.1016/j.jclinepi.2011.07.011.

60. Arain M, Campbell MJ, Cooper CL, Lancaster GA. What is a pilot or feasibility study? A review of current practice and editorial policy. BMC Med Res Methodol. 2010;10:1-7.

61. Arain M, Campbell M, Cooper C, Lancaster G. What is a pilot or feasibility study? A review of current practice and editorial policy. BMC Medical Research Methodology. 2010;10. https://doi.org/10.1186/1471-2288-10-67.

62. Hooper R. Justifying sample size for a feasibility study. Res Design Service. https://doi.org/10.3748/wjg.v12.i17.2661.

63. Lancaster GA, Dodd S, Williamson PR. Design and analysis of pilot studies: recommendations for good practice. J Eval Clin Pract. 2004;10:307-12

64. Suresh K. An overview of randomization techniques: an unbiased assessment of outcome in clinical research. J Hum Reprod Sci. 2011;4:8-11. https://doi.org/10.4103/0974-1208.82352.

65. Csikszentmihalyi M. The flow experience and its significance for human psychology. Optimal Experience: Psychological Studies of Flow in Consciousness. Cambridge: Cambridge University Press; 1988:15-35. https:// doi.org/10.1017/CBO9780511621956.002 .

66. Reynolds F, Prior S. Creative adventures and flow in art-making: a qualitative study of women living with cancer. $\mathrm{Br} J$ Occup Ther. 2006; 69:255-62.

67. Lawson LM, Cline J, French A, Ismael N. Patient perceptions of a 1-h artmaking experience during blood and marrow transplant treatment. Eur J Cancer Care (Engl). 2017;26. https://doi.org/10.1111/ecc.12482.

68. Fancourt D. Arts in health: designing and researching interventions. Oxford: Oxford University Press; 2017.

69. Lawson LM, Williams P, Glennon C, Carithers K, Andrejack A, Wright N. Effect of art making on cancer-related symptoms of blood and marrow transplantation recipients. Oncol Nurs Forum. 2012;39:407.

70. Bugge C, Williams B, Hagen S, Logan J, Glazener C, Pringle S, et al. A process for Decision-making after Pilot and feasibility Trials (ADePT): development following 
a feasibility study of a complex intervention for pelvic organ prolapse. Trials. 2013; 14:1-13. https://doi.org/10.1186/1745-6215-14-353.

71. Avery KNL, Williamson PR, Gamble C, O'Connell Francischetto E, Metcalfe C, Davidson $\mathrm{P}$, et al. Informing efficient randomised controlled trials: exploration of challenges in developing progression criteria for internal pilot studies. BMJ Open. 2017;7:e013537. https//doi.org/10.1136/bmjopen-2016-013537.

72. O'Cathain A, Hoddinott P, Lewin S, Thomas KJ, Young B, Adamson J, et al. Maximising the impact of qualitative research in feasibility studies for randomised controlled trials: guidance for researchers. Pilot Feasibility Stud. 2015;1:32. https://doi.org/10.1186/s40814-015-0026-y.

73. Kim Y, Evangelista LS, Park Y-G. Anxiolytic effects of music interventions in patients receiving incenter hemodialysis: a systematic review and meta-analysis. Nephrol Nurs J. 2015;42:339-48. https://doi.org/10.1007/ s10459-010-9231-X.

74. Loosman WL, Siegert CEH, Korzec A, Honig A. Validity of the Hospital Anxiety and Depression Scale and the Beck Depression Inventory for use in end-stage renal disease patients. Br J Clin Psychol. 2010;49:507-16. https:// doi.org/10.1348/014466509X477827.

75. Annunziata MA, Muzzatti B, Altoé G. Defining hospital anxiety and depression scale (HADS) structure by confirmatory factor analysis: a contribution to validation for oncological settings. Ann Oncol. 2011; 22:2330-3.

76. Glover C, Banks P, Carson A, Martin CR, Duffy T. Understanding and assessing the impact of end-stage renal disease on quality of life. Patient. 2011:4:19-30.

77. Pothoulaki M, Macdonald RAR, Flowers P, Stamataki E, Filiopoulos V, Stamatiadis D, et al. An investigation of the effects of music on anxiety and pain perception in patients undergoing haemodialysis treatment. J Health Psychol. 2008;13:912-20. https://doi.org/10.1177/1359105308095065.

78. Peterson JC, Pirraglia PA, Wells MT, Charlson ME. Attrition in longitudinal randomized controlled trials: home visits make a difference. BMC Med Res Methodol. 2012;12:1-12.

79. Zyoud SH, Daraghmeh DN, Mezyed DO, Khdeir RL, Sawafta MN, Ayaseh NA, et al. Factors affecting quality of life in patients on haemodialysis: a crosssectional study from Palestine. BMC Nephrol. 2016;17:44. https://doi.org/10. 1186/s12882-016-0257-z.

80. Eldridge SM, Chan CL, Campbell MJ, Bond CM, Hopewell S, Thabane L, et al. CONSORT 2010 statement: extension to randomised pilot and feasibility trials. Pilot Feasibility Stud. 2016;2:64. https://doi.org/10.1186/ s40814-016-0105-8.

81. Francis JJ, Johnston M, Robertson C, Glidewell L, Entwistle V, Eccles MP, et al. What is an adequate sample size? Operationalising data saturation for theory-based interview studies. Psychol Health. 2010;25:1229-45.

82. Schneider M, Hall W, Hernandez A, Hindes K, Montez G, Pham T, et al. Rationale, design and methods for process evaluation in the HEALTHY study. Int J Obes. 2009;33(Suppl 4):S60-7. https://doi.org/10.1038/ijo.2009.118.Rationale.

83. Guest G, Bunce A, Johnson L. How many interviews are enough? Field methods. 2006;18:59-82. https://doi.org/10.1177/1525822X05279903.

84. Carlsen B, Glenton C. What about N? A methodological study of sample-size reporting in focus group studies. BMC Med Res Methodol. 2011;11:26. https://doi.org/10.1186/1471-2288-11-26.

85. O'Cathain A, Thomas KJ, Drabble SJ, Rudolph A, Goode J, Hewison J. Maximising the value of combining qualitative research and randomised controlled trials in health research: the QUAlitative Research in Trials (QUART) study - a mixed methods study. Health Technol Assess (Rockv). 2014;18:1-197.

86. Green J, Thorogood N. Qualitative methods for health research. London: Sage Publications; 2004.

87. Forman J, Heisler M, Damschroder LJ, Kaselitz E, Kerr EA. Development and application of the RE-AIM QuEST mixed methods framework for program evaluation. Prev Med Reports. 2017;6:322-8. https://doi.org/10.1016/j.pmedr. 2017.04.002.

88. Braun V, Clarke V. Using thematic analysis in psychology. Qual Res Psychol. 2006;3:77-101. https://doi.org/10.1191/1478088706qp063oa.

89. Steinke I. Quality criteria in qualitative research. A companion to Qual Res. 2004:184-190

90. Hounsome N, Shearer J. What kind of health economic input is appropriate in a feasibility study? National Institute for Health Research. 2015. https:// www.rds-london.nihr.ac.uk/wpcms/wp-content/uploads/2018/09/Healtheconomic-input-in-a-feasbility-study.pdf. Accessed 26 Nov 2017.
91. Marques E, Johnson EC, Gooberman-Hill R, Blom AW, Noble S. Using resource use logs to reduce the amount of missing data in economic evaluations alongside trials. Value Heal. 2013;16:195-201. https://doi.org/10. 1016/j.jval.2012.09.008

92. National Institute for Health and Care Excellence. Assessing cost effectiveness | Guidance and guidelines | NICE. 2012. https:/www.nice.org.uk/process/pmg6/ chapter/assessing-cost-effectiveness. Accessed 26 Nov 2017.

93. Schneider S, Münte T, Rodriguez-Fornells A, Sailer M, Altenmüller E. Musicsupported training is more efficient than functional motor training for recovery of fine motor skills in stroke patients. Music Percept. 2010;27:271-80.

94. Hertrampf R-S, Warja M. The effect of creative arts therapy and arts medicine on psychological outcomes in women with breast or gynecological cancer: a systematic review of arts-based interventions. Arts Psychother. 2017;56:93-110. https://doi.org/10.1016/j.aip.2017.08.001.

95. Leckey J. The therapeutic effectiveness of creative activities on mental well-being: a systematic review of the literature. J Psychiatr Ment Health Nurs. 2011:18:501-9.

96. Erikson JM. The arts as healing. Am J Orthop. 1983;53:602-18.

97. Hunter R, Shearer J. Cost-consequences analysis - an underused method of economic evaluation. National Institute for Health Research. 2014. http:// www.rds-london.nihr.ac.uk/How-to-design-a-study-find-funding/Healtheconomics/Cost-consequences-analysis.aspx. Accessed 26 Nov 2017.

98. McConnell T, Graham-Wisener L, Regan J, McKeown M, Kirkwood J, Hughes $\mathrm{N}$, et al. Evaluation of the effectiveness of music therapy in improving the quality of life of palliative care patients: a randomised controlled pilot and feasibility study. BMJ Support Palliat Care. 2016;6:398. https://doi.org/10. 1136/bmjspcare-2016-001204.37.

99. Schulz KF, Grimes DA. Epidemiology series sample size slippages in randomised trials : exclusions and the lost and wayward. 2002;359:781-785.

100. Popp L, Schneider S. Attention placebo control in randomized controlled trials of psychosocial interventions: theory and practice. Trials. 2015;16:15-7.

\section{Ready to submit your research? Choose BMC and benefit from:}

- fast, convenient online submission

- thorough peer review by experienced researchers in your field

- rapid publication on acceptance

- support for research data, including large and complex data types

- gold Open Access which fosters wider collaboration and increased citations

- maximum visibility for your research: over $100 \mathrm{M}$ website views per year

At $\mathrm{BMC}$, research is always in progress.

Learn more biomedcentral.com/submissions 